\title{
Soziale Medien als politischer Informationskanal
}

\author{
Katrin Praprotnik ${ }^{1, *}$, Flooh Perlot², Daniela Ingruber ${ }^{1}$, Peter Filzmaier ${ }^{1}$ \\ 1 Donau-Universität Krems \\ 2 Universität Graz \\ * katrin.praprotnik@donau-uni.ac.at
}

\section{Zusammenfassung}

Ein lebendiger politischer Diskurs ist ein wesentlicher Bestandteil einer funktionierenden Demokratie. Mit steigenden Nutzerzahlen der sozialen Medien besitzt auch die politische Diskussion, die über diese Plattformen geführt wird, eine zunehmende Bedeutung. Die vorliegende Studie untersucht deshalb die KonsumentInnen von politischer Information über soziale Netzwerke anhand des wenig untersuchten Fallbeispiels Österreich. Unsere Modelle basieren auf einer Sekundärdatenauswertung der Studie Digitalmonitor ( $\mathrm{N}=\mathrm{I} .2 \mathrm{OO})$. Die Ergebnisse zeigen, dass die NutzerInnen von politischer Information über soziale Netzwerke unter anderem politisch stark interessiert, auf einer Links-Rechts-Skala eher extrem eingestellt sind und ein geringes Vertrauen in traditionelle Medien haben. Der neue Informationskanal sorgt nicht zwangsläufig für einen gleichberechtigteren Zugang zu Information. Für Menschen, die mit dem bisherigen Angebot nicht zufrieden waren, bietet er jedoch eine Alternative.

\section{Schlüsselwörter}

Soziale Medien, politische Information, Mediennutzungsverhalten, Vertrauen in Politik, quantitative Analyse, Umfrage

\section{Social Media as Information Channel}

\begin{abstract}
A vivid political discourse is a crucial component of a functioning democracy. Since user numbers of social networks are increasing, the political debate via these channels becomes more important. Therefore, the present study analyses the consumers of political information through social networks, using the case of Austria as an example. The models are based upon a secondary data analysis of the Digitalmonitor $(\mathrm{N}=\mathrm{I} .2 \mathrm{OO})$. Our results show that social media consumers of political information are, among other things, highly interested in politics, hold rather extreme values on a political left-right scale and have little trust in traditional media channels. We conclude that social media do not guarantee equal access to information. However, for people dissatisfied with the traditional media, it provides an alternative.
\end{abstract}

\section{Keywords}

Social media, political information, media use, trust in politics, quantitative analysis, questionnaire

The authors have declared that no competing interests exist. 


\section{Einleitung}

Das Mediennutzungsverhalten der letzten Dekaden wird in zahllosen Studien und Artikeln beschrieben (etwa Flusser 1987, Sarcinelli I998, Aarts/Semetko 2003), durchaus mit einigen Gemeinsamkeiten. Sobald ein neues Medium mit Massenpotential auftaucht, sei es Radio, Kino, Fernsehen oder Internet, wird ihm zuerst die Chance auf mehr Gleichheit und einfacheren Zugang zu (politischer) Information nachgesagt (vgl. dazu den Begriff des technoromanticism; Coyne 200I). Anschließend gibt es eine Phase, die den zivilisatorischen ebenso wie den politischen Niedergang unterstellt, und letztlich zeigt sich, dass jegliche Vor- und Nachteile hauptsächlich mit den jeweils spezifischen Nutzungsgewohnheiten einhergehen. Wie Personen Informationen suchen und wie sie sich entscheiden, welche Informationen sie sich woher holen, hat mit Erfahrungen, Gewohnheiten, vor allem aber auch mit der eigenen Persönlichkeit zu tun (Wolling 20I6, 25). So nutzen viele Menschen zwar dieselben Medien, doch nicht unbedingt aus den gleichen Gründen. Was für die einen hauptsächlich ein Unterhaltungsmedium darstellt, ist für die anderen eine essentielle Quelle politischer Information (vgl. Hughes/ Rowe et al. 20II, 562; Sheldon/Bryant 2016, 90 ff.).

Voraussichtlich trifft dies auch auf die Sozialen Medien wie Facebook oder Twitter zu. Vergleicht man verschiedene Studien, befindet man sich aktuell in einer Zwischenphase, in der nicht mehr ganz so euphorisch von einer völligen Veränderung der Politik aufgrund neuer Medien gesprochen wird (Kneuer/Salzborn 2016; Dickel/ Schrape 20I5). Dabei fällt außerdem auf, dass ein Großteil dieser Studien einen Fokus auf den englischsprachigen und hier vor allem auf den US-amerikanischen Raum legt. Arbeiten, die sich auf europäische Staaten konzentrieren, sind bis auf wenige Ausnahmen rar (vgl. für Deutschland Faas/Sack 20I6; Hagen et al. 20I7). Es ist aber keineswegs klar, dass die Ergebnisse aus nordamerikanischen Studien auf europäische Länder wie Österreich übertragbar sind. Insbesondere die Unterschiede im Parteiensystem sind evident: Während sich in den USA mit den Republikanern und Demokraten zwei große Blöcke gegenüberstehen, gibt es in den meisten europäischen Ländern deutlich mehr relevante Akteure.

Der Raum für neue Forschung im Bereich Mediennutzungsverhalten sozialer Netze zur politischen Information ist damit gegeben. Die Relevanz solcher Arbeiten ist ebenfalls vorhanden. Ein zentrales Element demokratischer Systeme ist ein freier Diskurs über die politische Gestaltung des Landes. Soziale Medien bieten einen weiteren Raum für einen solchen Austausch, da sie Individuen eine Plattform stellen, sich mit anderen Menschen zu vernetzen und zu diskutieren. Gerade weil es in der politischen Kommunikation wohl nicht zu einer Revolution durch einen nun gleichberechtigten Zugang $\mathrm{zu}$ Information für alle kommen wird, ist es wesentlich zu untersuchen, wer soziale Plattformen als Nachrichtenquelle nützt und was diese Nutzung erklärt.

Im Mittelpunkt der vorliegenden Studie stehen daher folgende Fragen: Welche Akteure nützen soziale Medien zur politischen Information? Und: Welche Rolle spielt Vertrauen bei der Nutzung sozialer Medien zur politischen Information? Wir analysieren sowohl die aktive Suche nach derartigen Informationen als auch die passive oder zufällige Aufnahme von politischen Neuigkeiten über soziale Plattformen. Wir untersuchen hingegen nicht die SenderInnen dieser Informationen und auch nicht mögliche Effekte, die sich aus dem Nachrichtenkonsum ergeben. Wir ergänzen die nationale und internationale Literatur insbesondere durch unseren Fokus auf das Fallbeispiel Österreich. Nach bestem Wissen der AutorInnen liegt für Österreich bislang nur eine Forschungsnotiz zu diesem Thema vor, deren $\mathrm{Da}-$ tengrundlage bereits aus dem Jahr 2010 stammt (Vonbun/Schönbach 2014). Außerdem ist Österreich Teil einer in dieser Hinsicht relevanten Untersuchung der Eurobarometerdaten (eine der wenigen Analysen im europäischen Raum; Wolling 2016), wobei die Ergebnisse zu Österreich nicht gesondert diskutiert werden. In der vorliegenden Arbeit analysieren wir einen Datensatz aus einer quantitativen Befragung von rund I.200 Personen. Schließlich beleuchten wir mit dem zusätzlichen Fokus auf den Einfluss von Vertrauen einen bislang wenig beachteten Aspekt.

\section{Theorie}

Im Mittelpunkt unseres Forschungsinteresses stehen der einzelne Bürger und die einzelne Bürgerin. Während sich diese bemühen, rational $\mathrm{zu}$ agieren, und dies auch gemäß ihrer Ressourcen umsetzen (Downs I957), werden Lesarten der wahrgenommenen Realität von persönlichen Erfahrungen, Emotionen und nicht zuletzt äußerlichen Umständen (dazu zählen sowohl Strukturen als auch Ideologien) beeinflusst (Fairclough 200I). Wenn es um Mediennutzung geht, kommen zudem der Zeitfaktor, Medienkonsumgewohnheiten sowie der Einfluss durch das unmittelbare Umfeld hinzu (Johnson/ Milani 20IO; Barthes I980), des Weiteren der Wunsch, sich mit Hilfe der Medien eine Welt kreieren zu lassen (Baudrillard I983 und 2003; Žižek 1997). Diese Grundannahmen bestimmen die weitere Herleitung unserer Hypothesen in zwei Gruppen. Die erste Gruppe stellt die Hypothesen mit einem Fokus auf die Akteure vor. Wir diskutieren die Eigenschaften der BürgerInnen, die es wahrscheinlich erscheinen lassen, dass soziale Medien zur politischen Information genützt werden. Die zweite Gruppe stellt die Hypothesen mit Fokus auf Vertrauen vor. Hier geht es um die Frage, welche Rolle Vertrauen 
in traditionelle sowie moderne Medien bei der Nutzung sozialer Dienste spielt.

\section{Fokus: Akteure}

Als Hintergrund für den Fokus auf die Akteure lohnt sich zunächst ein Blick auf die vorhandenen deskriptiven Daten zur Internet- und Social Media-Nutzung im Kontext der Informationsmedien in Österreich. 86 Prozent der österreichischen Bevölkerung ab I4 Jahren gelten generell als InternetnutzerInnen, rund 70 Prozent als (fast) tägliche OnlinerInnen (Integral 20I7). Trotz der starken Verbreitung zieht sich nach wie vor eine Alterskluft durch die soziodemographische Struktur der Menschen online, sodass ältere Menschen auch seltener das Internet nützen. Unterschiede zwischen Männern und Frauen sind demgegenüber nur schwach ausgeprägt. Nach formaler Bildung sind Personen mit höheren Bildungsabschlüssen (Matura oder höher) etwas häufiger im Internet aktiv (Statistik Austria 2018a; Integral 20I7).

Soziale Netzwerke stellen eine Unterkategorie spezieller Dienste im Internet dar, die von rund 6o Prozent der ÖsterreicherInnen (I6 bis 74 Jahre) genutzt werden (Eurostat 2018). Das Alter bildet auch hier einen wesentlichen Faktor: Während bei den unter 25-Jährigen 80 Prozent praktisch jeden Tag soziale Netzwerke verwenden, sind es bei Personen ab 55 Jahren nur elf Prozent. Ähnlich, wenn auch nicht ganz so deutlich, wirkt sich eine höhere formale Bildung aus (Personen mit einem Bildungsabschluss nach Erreichen des 20. Lebensjahres nutzen Social Media zu 42 Prozent täglich, jene mit einem Bildungsabschluss im Alter von I5 Jahren nur zu I4 Prozent), das Geschlecht spielt hingegen keine Rolle (Europäische Kommission 20I7, 20).

Diese Daten unterscheiden zunächst nicht nach der jeweiligen Motivation, das Internet zu nutzen. Doch auch bei der Suche nach politischer Information im Netz scheinen die Variablen Alter und formale Bildung bestimmend und die Unterschiede zwischen den $\mathrm{Ge}-$ schlechtern gering zu sein (Reuters Institute 20I7, II; Europäische Kommission 20I7, 40).

Angesichts der bereits vorhandenen Erkenntnisse dient Hypothese I in erster Linie dazu, den vorliegenden Forschungsstand anhand aktueller Daten zu überprüfen. Wir erwarten daher auch in den multivariaten Modellen ähnliche soziodemographische Merkmale bei den NutzerInnen, Hypothese I lautet:

HI: Personen, die soziale Medien zur politischen Information verwenden, weisen folgende soziodemographische Merkmale auf:

- Hra: Je jünger eine Person ist, umso wahrscheinlicher ist die Nutzung sozialer Medien zur politischen Information.
- Hib: Je formal besser gebildet eine Person ist, umso wahrscheinlicher ist die Nutzung sozialer Medien zur politischen Information.

- Hic: Männliche und weibliche Personen nützen soziale Medien zur politischen Information gleichermaßen.

Nicht nur soziodemographische Merkmale, sondern auch das persönliche Interesse können für eine Nutzung sozialer Medien zur politischen Information verantwortlich sein. Wie oben beschrieben ist die Zeit, die für den Medienkonsum zur Verfügung steht, begrenzt und BürgerInnen müssen gemäß den eigenen Präferenzen jene Inhalte auswählen, von denen sie sich den größten Nutzen versprechen (Johnson/Milani 20IO). Wenn BürgerInnen Interesse am politischen Geschehen hegen, wird der Einsatz eines weiteren Kanals für sie einen Nutzen generieren und die Wahrscheinlichkeit, dass derartige Plattformen zur Information eingesetzt werden, steigt. In diese Richtung deuten auch Daten von Vonbun/Schönbach (20I4, 204), die zeigen, dass das politische Interesse ein wesentlicher Erklärungsfaktor für die politische Verwendung sozialer Netzwerke in Österreich darstellt. Und auch die wenigen europäischen Studien gehen von einem Zusammenhang zwischen politischem Interesse und Medienkonsum aus (vgl. etwa für Deutschland: Emmer et al. 20II; für Frankreich: Jouët et al. 2oII und für Schweden: Östman 20I2). Ein entsprechender Einfluss des persönlichen Interesses wird in Hypothese 2 formuliert:

$\mathrm{H} 2$ : Je größer das politische Interesse, umso wahrscheinlicher ist die Nutzung sozialer Medien zur politischen Information.

Die Überprüfung dieser Hypothese ist auch für die Beurteilung sozialer Medien aus einer demokratiepolitischen Perspektive relevant. Ist das politische Interesse des Einzelnen ausschlaggebend für die Verwendung sozialer Plattformen zur politischen Information, so schränkt das den Beitrag dieser Kanäle zu einer Ausweitung des Publikums für politische Information bzw. der Teilnehmenden am politischen Diskurs ein. Teil der These ist somit, dass neue NutzerInnen demnach nur gewonnen werden, sofern diese ebenfalls bereits politisch interessiert sind.

Die vorhandene Literatur fokussiert bislang vor allem auf die Frage, welche Effekte die Nutzung sozialer Medien auf bereits vorhandene oder sich entwickelnde politische Polarisierung, hin zu politisch extremen Positionen, besitzt (vgl. Barberá 2015; Faas/Sack 2016; Schrape 2015; Hagen et al. 2017). Ein bislang wenig beachteter Aspekt ist, dass möglicherweise bereits bei der Nutzungsentscheidung eine verzerrte Auswahl stattfindet, zum Teil durch algorithmische Selektion - die technische Vorauswahl von Inhalten basierend auf vorlie- 
genden Daten zur/zum NutzerIn und deren/dessen Interessen - zuweilen aber auch unbeabsichtigt (Hagen et al 20I7b), und sich Personen mit extremen Ansichten eher sozialen Medien zur politischen Information zuwenden, weil sie dort eine Welt vorfinden, die sie sich quasi selbst (bewusst und unbewusst) zusammenstellen (Hagen et al 2017 a und b).

Das dazu passende Schlagwort ist jenes einer Echokammer oder einer Filterblase, in der man Seinesgleichen trifft und zu sehen bekommt, was man sich wünscht (Faas/Sack 2016, 4I; Schrape 2015, 8; Hagen et al. 2017, 3; zum Begriff vgl. Pariser 2012). Diese These hat weitreichende Folgen, da sie eine (mehr oder weniger starke) Verzerrung von Informationen durch die Nutzung von Social Media als Quellen plausibel erscheinen lässt. Die Befunde zu diesem Thema sind allerdings ambivalent und stellen in erster Linie die Frage, welche Effekte soziale Netzwerke auf die NutzerInnen haben.

So kommt Barberá zum Schluss, dass Social Media die politische Polarisierung durchaus verringern können, da sie Kontakte über das engste persönliche Umfeld hinaus ermöglichen und somit auch eine Konfrontation mit neuen oder anderen Meinungen (2015). Besonders eine Nutzung dieser Kanäle zur politischen Informationsbeschaffung würde zur Entpolarisierung beitragen (Barberá et al. 2015, 7 und 9). Während er aus seinen Daten einen geringeren Einfluss der Echokammern auf Liberale bzw. sich selbst eher links einordnende Personen folgert, sehen Dubois/Blank die politischen Ränder rechts wie links gleichermaßen betroffen (2018, II). Sie halten ebenfalls jene für weniger anfällig, die unterschiedliche Medien konsumieren (2018, I2).

Zum selben Ergebnis kommen Colleoni et al. (2014, 326) in Bezug auf die US-DemokratInnen, allerdings ziehen sie daraus den Schluss, dass Homophilie in den Social Media zur Polarisierung beitrage $(2014,328)$. Was die Beeinflussung betrifft, beurteilen Diehl et al. wiederum, dass jeglicher Kontakt über Social Media letztlich politisch ist oder zu politischen Diskussionen führt, zuweilen indirekt (2015, I4 f.). In diesem Zusammenhang sagen Hagen et al., dass Extrempositionen mit zunehmender Nachrichtennutzung nachlassen (20I7, IO).

Chang et al. wiederum stellen fest, dass UserInnen in den Social Media zwar mit heterogeneren Meinungen in Kontakt kommen als in den Massenmedien, dies jedoch nicht unbedingt zu mehr politischen Debatten in den Social Media führe, weil man die ungeliebten Meinungen von FreundInnen, KollegInnen oder Verwandten häufig unkommentiert stehen lasse, um Konflikte zu vermeiden (2017, 289).

Boxell et al. weisen für die USA darauf hin, dass ausgerechnet jene Gruppen, die am wenigsten wahrscheinlich Social Media nützen, etwa die +65 -Jährigen, eine drei Mal so hohe Polarisierung zeigen. Diese fände demnach an anderer Stelle statt (2OI8; $4 \mathrm{f}$.).
Die umgekehrte Frage, nämlich inwieweit soziale Netzwerke gerade im Kontext politischer Information vermehrt Personen anziehen, die von vornherein bereits eine stärker polarisierte politische Haltung einnehmen, wird deutlich seltener gestellt. Vonbun/Schönbach (20I4) finden einen entsprechenden leichten Effekt in ihrer Analyse, wonach politisch stark rechts orientierte NutzerInnen etwas häufiger am politischen Diskurs online teilnehmen.

Wir fokussieren uns auf diese Perspektive und knüpfen mit Hypothese 3 daran an:

H3: Je näher sich eine Person im politischen LinksRechts Spektrum an den Rändern verortet, umso wahrscheinlicher ist die Nutzung sozialer Medien zur politischen Information.

\section{Fokus: Vertrauen}

Eine relevante Perspektive für den Erklärungsversuch der Social Media-Nutzung im politischen Informationskontext stellen die Einstellungen zu Medien generell dar, die als eine Art Vertrauen beschrieben werden können. Dabei geht es sowohl um das Vertrauen in die traditionellen Massenmedien und das Internet, als auch um konkrete Erfahrungen mit den sozialen Netzwerken. Generell wird sozialen Netzwerken in geringerem Umfang Vertrauen entgegengebracht als Fernsehen, Radio oder Tageszeitungen (vgl. u.a. Reuters 2015, II; für Deutschland Faas/Sack 2016, 23). Eine Rolle dafür spielt mitunter der nach wie vor vorhandene Fokus von Social Media auf Unterhaltung (Faas/Sack 2016, 32).

Das Vertrauen, das den traditionellen Medien entgegengebracht wird, besitzt in Österreich besondere Relevanz. Das heimische Mediensystem ist von wenigen Unternehmen und einer eingeschränkten Vielfalt geprägt (Plasser/Lengauer 2012, 30 f.). Die tagesaktuelle Printmedienlandschaft ist mit aktuell i4 Titeln sehr überschaubar. In Radio und Fernsehen dominieren nach wie vor die Programme des ORF (im Informationsbereich noch stärker als generell), private Anbieter haben (im Radiobereich) überwiegend einen Hintergrund in Tageszeitungsverlagen oder sind (im Fernsehen) an deutsche Sendergruppen (Pro7/SatI) angeschlossen. Die begrenzte Anzahl an Alternativen im Sektor traditioneller Medien kann damit im Falle einer skeptischen bis kritischen Haltung diesen gegenüber die sozialen Medien als Informationsquelle noch attraktiver machen (vgl. Vonbun/Schönbach 20I4, 20I).

Hypothese 4 formuliert daher wie folgt:

$\mathrm{H}_{4}$ : Je geringer das Vertrauen in traditionelle Medien ist, umso wahrscheinlicher ist die Nutzung sozialer Medien zur politischen Information. 
Die Überprüfung dieser Hypothese gibt Aufschluss darüber, ob soziale Medien ersetzend von oder ergänzend zu den traditionellen Medien konsumiert werden. Wenn die Daten den in $\mathrm{H}_{4}$ formulierten Zusammenhang unterstützen, dann deutet das darauf hin, dass die sozialen Netzwerke als Ersatz dienen, der es den NutzerInnen erlaubt, Informationen von einem alternativen Sender zu erhalten. Finden wir hingegen keine Anzeichen für $\mathrm{H}_{4}$, dann werden soziale Plattformen als zusätzlicher Kanal für den eigenen Medienkonsum verwendet. Das würde auch die Entstehung paralleler Kommunikationswelten weniger wahrscheinlich erscheinen lassen.

Im nächsten Schritt möchten wir das Gefühl des Vertrauens und seine Auswirkungen auf die Mediennutzung weiter spezifizieren. Ein geringes Vertrauen in die traditionellen Medien mag dazu führen, dass vermehrt soziale Medien genutzt werden. Gleichzeitig scheint es relevant, dass auch jenem Kanal, der die Informationen aus den sozialen Netzwerken transportiert, Vertrauen entgegengebracht wird. Hypothese 5 geht daher davon aus, dass auch das Vertrauen in das Internet eine wesentliche Erklärungskraft für den Einsatz sozialer Netze zur Information über Politik besitzt.

H5: Je größer das Vertrauen in das Internet ist, umso wahrscheinlicher ist die Nutzung sozialer Medien zur politischen Information.

Neben unbestimmten Gefühlen des Misstrauens in traditionelle Medien oder des Vertrauens in das Internet können aber auch konkrete Erfahrungen die Einstellung zu sozialen Diensten beeinflussen. Spätestens hier drängt sich das Thema "Fake News“ auf. Social Media eignen sich aufgrund ihrer Struktur und der variablen AutorInnenschaften bestens für Fake News. Wie Allcott und Gentzkow zeigen konnten, waren Fake News während des US-Präsidentschaftswahlkampfs 2016 tatsächlich weit verbreitet - und doch nur zu einem geringen Teil mitverantwortlich für den Wahlausgang (Allcott/ Gentzkow 2017, 232). Die eigentliche Gefahr von Fake News dürfte sein, dass Vertrauen, Wahrheit und Meinung miteinander vermischt werden (Palmer 2018). Wir gehen schließlich in $\mathrm{H} 6$ davon aus, dass schlechte Erfahrungen die NutzerInnen von dem neuen Medium abschrecken und der Nachrichtenkonsum dadurch weniger wahrscheinlich wird.

H6: Wenn die betroffene Person noch keine Erfahrung mit "Fake News" (Falschnachrichten) gemacht hat, wird die Nutzung sozialer Medien zur politischen Information wahrscheinlicher.

\section{Methode}

Die vorgenommene Analyse baut auf einer Sekundärdatenauswertung der Studie "Digitalmonitor" auf, die das Institut für Strategieanalysen (ISA) im Jahr $2017 \mathrm{im}$ Auftrag des damaligen Staatssekretariats für Diversität, öffentlichen Dienst und Digitalisierung durchgeführt hat (ISA 2017).

Dabei handelte es sich um eine Online-Befragung (CAWI) unter I.2O4 Personen, die anhand eines Quotenplans aus einem bestehenden Panel eines Marktforschungsinstituts ausgewählt wurden, wobei aus Gründen des ursprünglichen Studieninteresses 200 I4- bis I8-Jährige als fixe Gruppe befragt wurden. ${ }^{.}$Die Grundgesamtheit stellte die internetaktive Bevölkerung zwischen I4 und 70 Jahren dar, die Struktur der Quotenstichprobe wurde anhand der Variablen Geschlecht, Alter und formale Bildung an sie angenähert (siehe Anhang A.).

Die Stichprobe spiegelt das Geschlechterverhältnis der Grundgesamtheit wider, weicht aber bei der formalen Bildung leicht ab, sie enthält hier mehr Personen mit Matura, jedoch weniger mit Lehrabschluss oder akademischer Ausbildung. Nach dem Alter ergeben sich Unterschiede durch die fixe Quote der Befragten unter I8 Jahren. Die Struktur der Stichprobe wurde für die Auswertung entsprechend gewichtet, um die Differenzen etwas auszugleichen. Die Feldzeit dauerte von 22. Mai bis 9. Juni 2017.

Inhaltlich drehte sich die Erhebung um das Mediennutzungsverhalten der Befragten, konkret mit einem Fokus auf soziale Netzwerke und einem Vergleich traditioneller und Online-Medien. Dazu kamen Befragungsteile zu Themen wie Fake News, Cybermobbing und Hasspostings. Die Ergebnisse der Studie wurden bislang lediglich deskriptiv präsentiert.

Die vorliegende Arbeit präsentiert eine tiefergehende und erstmals multivariate Analyse der vorhandenen Daten. Im Folgenden werden die abhängigen sowie die unabhängigen Variablen, die in der Analyse verwendet werden, im Detail beschrieben.

Die abhängige Variable der Analyse basiert auf der Frage "Woher beziehen Sie Ihre Informationen über politische Themen in Österreich?". 2 Die Befragten konnten aus einer Liste die Medien Fernsehen, Radio, Tageszeitungen, Internet ohne soziale Netzwerke und soziale

I Unter dem Begriff „Panel“ ist ein bestehender Pool an Personen zu verstehen, auf den für Befragungen immer wieder zugegriffen werden kann. Das genutzte Panel bestand zum damaligen Zeitpunkt aus rund 30.000 Personen (meinungsraum.at 2018).

2 Bei Personen, die bei der vorangestellten Filterfrage „Interessieren Sie sich für politische Themen in Österreich?" angegeben haben, sich eher nicht für politische Fragen zu interessieren, wurde dieser Frage folgender Zusatz vorangestellt: „Wenn Sie sich doch einmal informieren möchten $(\ldots)^{\star}$. Diese und alle weiteren in der Studie benutzten Fragen befinden sich im genauen Wortlaut im Anhang B. 
Netzwerke auswählen, wobei Mehrfachantworten möglich waren. Auf Grundlage der Antworten wurden zwei Gruppen gebildet: Personen, die soziale Netzwerke als eine Quelle für Informationen über politische Themen nannten $(\mathrm{n}=377)$, und Personen, die dies nicht taten $(\mathrm{n}=774)$.

Die Nennung von sozialen Netzwerken (oder anderen Medien) wurde als bewusste und zielgerichtete Entscheidung für diese Informationsquelle interpretiert und bedeutet nicht, dass nur diese Personen soziale Medien nutzen oder über diesen Dienst politische Nachrichten zugetragen bekommen. Das zeigt sich vor allem daran, dass die Zahl der generellen NutzerInnen sozialer Netzwerke deutlich höher ist $(\mathrm{n}=967)$.

Die Hypothesen zur Überprüfung des Akteurs in den sozialen Netzwerken auf der Suche nach politischer Information benötigen Angaben zum Alter, dem Bildungsgrad, dem Geschlecht (HI), dem politischen Interesse der Befragten (H2) sowie eine Einordnung auf der politischen Links-Rechts-Skala (H3). Die kontinuierliche Variable Alter in Jahren gibt, wie der Name bereits sagt, das Alter der befragten Personen in Jahren wieder. Die kategoriale Variable formaler Bildungsgrad unterscheidet die Bildungsabschlüsse Pflichtschule, Lehre, Berufliche Mittelschule/Fachschule, Matura und Universität/ Fachhochschule. Die binäre Variable Frau unterscheidet zwischen männlichen und weiblichen Probanden und nimmt im letzteren Fall den Wert I an. Die kontinuierliche Variable Interesse an der Politik dient der Überprüfung von Hypothese 2. Die Personen wurden gefragt, ob sie sich für politische Themen in Österreich interessieren. Die vierstufige Antwortskala unterscheidet zwischen ja, sehr / ja, eher schon / nein, eher nicht und nein, gar nicht. Die kontinuierliche Variable Politisch Extrem basiert schließlich auf der Links-Rechts-Einstufung der ProbandInnen auf einer Io-teiligen Skala von I (=sehr weit links) bis Io (=sehr weit rechts). Im Modell haben wir jeweils die äußeren zwei Werte zusammengefasst, sodass die Positionen in der Mitte (5 und 6) den Wert I zugeordnet bekommen haben und die politischen Extrempositionen (I und Io) den Wert 5. Das Ergebnis ist eine nunmehr 5-stufige Skala von I (=Politische Mitte) bis 5 (=Politisch Extrem). Eine derartige Umkodierung erlaubt den direkten Test von $\mathrm{H} 3$, die besagt, dass mit zunehmender Positionierung am politischen Rand eine zunehmende Nutzung sozialer Plattformen zur politischen Information erfolgt.

Die Hypothesen zur Rolle des Vertrauens bei der politischen Nutzung sozialer Medien werden anhand der folgenden Variablen geprüft: Die kontinuierliche Variable Vertrauen in traditionelle Medien basiert auf einer Fragebatterie, in der die ProbandInnen gebeten wurden, jeweils ihr Vertrauen in Zeitungen/Zeitschriften, Fernsehnachrichten und Radionachrichten im Vergleich zu sozialen Netzwerken auf einer Skala von I (hohes Ver- trauen in traditionelle Medien) bis 7 (hohes Vertrauen in soziale Netzwerke) einzustufen. Die Antworten auf diese drei Fragen wurden jeweils addiert und durch drei dividiert. ${ }^{3}$ Das Ergebnis ist eine kombinierte Messung des Vertrauens der ProbandInnen. Für eine kohärente Interpretation der Ergebnisse wurden die Werte umkodiert, sodass höhere Skalenwerte mit einem höheren Vertrauen in traditionelle Medien verbunden sind. Diese Frage wurde allerdings nur Personen gestellt, die angaben, zumindest aus einem sozialen Netzwerk auch politische Informationen zugetragen zu bekommen. ${ }^{4} \mathrm{Da}-$ durch verkleinert sich das Sample, das für einen Test der Hypothese 4 zum Vertrauen in die traditionellen Medien herangezogen werden kann. Es enthält nur mehr Personen, die zumindest passiv politische Information über soziale Medien erhalten. Damit ist jene Gruppe an Befragten gemeint, die soziale Netzwerke persönlich zwar nicht gezielt als Informationsquelle nutzen, dort aber gleichsam unabsichtlich mit politischen Informationen in Kontakt kommen. Hypothese 4 prüft daher für die Gruppe an zumindest passiven NutzerInnen politischer Information in sozialen Netzwerken, ob jene Menschen, die weniger Vertrauen in traditionelle Medien haben, soziale Netzwerke eher zur aktiven Information verwenden.

Die kontinuierliche Variable Vertrauen in Informationen aus dem Internet kombiniert ebenfalls die Zustimmung zu zwei Aussagen, die den Umgang mit OnlineNachrichten betreffen. Die erste Aussage fragte nach der Zustimmung zu dem Statement, dass man Informationen aus dem Internet nicht trauen kann. Die zweite beinhaltete die Behauptung, dass man sich häufig Gedanken über die Richtigkeit von Online-Informationen macht. Die Befragten konnten auf einer vierstufigen Skala Zustimmung beziehungsweise Ablehnung signalisieren. Wieder wurden die beiden Werte addiert und durch die Anzahl der gültigen Antworten dividiert. Das Ergebnis ist erneut ein metrischer Index zum Vertrauen in Online-Nachrichten. Letztlich gibt die kategoriale Variable Erfahrung mit Fake News, wie der Name bereits sagt, an, ob die befragte Person bereits einmal in Berührung mit Falschnachrichten gekommen ist. Das Antwortverhalten wird in vier Kategorien, die zwischen ja, sehr oft /

3 Nicht immer wurden alle drei Fragen durch die ProbandInnen beantwortet. Um nicht den kompletten Fall aus der Analyse zu verlieren, wurde die Anzahl der gültigen Antworten bei der Berechnung berücksichtig. Waren nur zwei Antworten gültig, so wurde die Summe auch nur durch zwei dividiert. Bei einer gültigen Antwort wurde dieser Wert verwendet. Die hohe Korrelation der Antworten zwischen den traditionellen Medien $(\mathrm{r}=0,7-0,8)$ rechtfertigt diesen Schritt.

4 Der genaue Fragewortlaut war: „Erhalten Sie über die Dienste auch Informationen über politische Themen in Österreich?" Die Frage folgte auf eine Abfrage der genutzten sozialen Netzwerke und wurde für jedes dieser Netzwerke gestellt. Wenn die Frage zumindest in einem Fall bejaht wurde, erhielt die Person die angesprochene Vergleichsfrage zwischen traditionellen Medien und Social Media. 
ja, manchmal / ja, aber nur vereinzelt und nein, nie unterscheiden, erfasst.

\section{Analyse}

Am Beginn dieses Artikels haben wir unsere forschungsleitenden Fragestellungen präsentiert. Wir wollten wissen: Wer nützt soziale Medien zur politischen Information? Und welche Rolle spielt dabei das Gefühl des Vertrauens? Zur Beantwortung dieser Forschungsfragen präsentiert Tabelle I nun drei binäre Logit-Modelle. Alle drei Modelle erklären dieselbe abhängige Variable. Diese gibt an, ob die befragte Person soziale Netzwerke zur Information über politische Themen in Österreich verwendet. Modell I widmet sich dem ersten Frageteil und testet die Hypothesen zum Akteur in den sozialen Medien. Modell II konzentriert sich hingegen ausschließlich auf die Annahmen zur Rolle von Vertrauen bei der politischen Informationssuche auf sozialen Plattformen. Schließlich verbindet Modell III beide Variablengruppen und überprüft, welche Faktoren in einem kombinierten Modell eine stabile Erklärungskraft besitzen. Die Koeffizienten sind in Odds Ratios (Chancenverhältnissen) angegeben. Das bedeutet, dass Werte über I auf eine steigende und Werte zwischen o und I auf eine sinkende Chance der politischen Nutzung sozialer Netzwerke hinweisen. Im Folgenden werden wir jeweils nur die Richtung des Effekts inklusive der Signifikanz der Koeffizienten direkt aus der Tabelle ablesen. Die Interpretation der Größe des Effekts erfolgt in einem zweiten Schritt auf Basis von vorhergesagten Wahrscheinlichkeiten für die Social Media-Nutzung, da jene anhand von Chancenverhältnissen nur wenig intuitiv ist. Eine erweiterte Diskussion der Odds Ratios finden Interessierte in den jeweiligen Fußnoten.

\section{Akteure der politischen Social Media-Nutzung}

Hypothese I orientierte sich am vorhandenen Forschungsstand zur Nutzung sozialer Netzwerke. Eswurde angenommen, dass die Wahrscheinlichkeit der Nutzung sozialer Netzwerke mit sinkendem Alter der Befragten (Hia) und höherer Bildung (Hib) steigt und sich nicht zwischen Männern und Frauen (HIc) unterscheidet.

Sowohl das schlanke Modell I als auch das gesamte Modell III bestätigen die erste Hypothese mit all ihren Spezifikationen und decken sich mit bisher bekannten deskriptiven Ergebnissen (Reuters Institute 20I7, II; Europäische Kommission 2016, 40). Die Variablen Alter in Jahren und formaler Bildungsgrad sind in beiden Fällen signifikant (mit Ausnahme der Ausprägung Lehre und Matura in Modell I bzw. BMS/Fachschule in Modell III). Das Odds Ratio kleiner I bei der Altersvariable deutet auf einen negativen, jene über I in den Zeilen der Bildungsab- schlüsse auf einen positiven Zusammenhang hin. Keinen Effekt zeigt, wie angenommen, das Geschlecht der befragten Personen (Frau). ${ }^{5}$

Wie verändert sich nun die Wahrscheinlichkeit, dass eine Person soziale Plattformen verwendet, um sich über das politische Geschehen in Österreich zu informieren, mit zunehmendem Alter beziehungsweise höherer formaler Bildung? Eine durchschnittliche Person im Alter von I6 Jahren verwendet mit einer Wahrscheinlichkeit von 0,65 soziale Medien zur Informationssuche. ${ }^{6}$ Dieser Wert sinkt bei um Io Jahre älteren Personen auf 0,58 und bei einer Person im Alter von 66 Jahren auf nur mehr o,3. Ebenfalls einen sehr starken Effekt sehen wir bei der Variablen formaler Bildungsgrad. Die Wahrscheinlichkeit der Nutzung steigt von o,39 im Falle eines Pflichtschulabschlusses auf 0,59 im Falle eines Universitäts- oder Fachhochschulabschlusses.

Hypothese 2 befasste sich auch mit dem Akteur und stellte die Behauptung auf, dass besonders politisch interessierte Menschen auch in sozialen Netzwerken Informationen über die nationale Politik suchen. Sie wird in den Modellen bestätigt. In Modell I nimmt die Variable Interesse an der Politik einen Wert von I,54 und in Modell III von I,42 an. Die Koeffizienten sind jeweils statistisch signifikant. Das bedeutet, dass die Wahrscheinlichkeit sich über soziale Medien politisch zu informieren bei Personen, die sich gar nicht für Politik interessieren, bei 0,26 liegt und bei Personen, die ein großes Interesse äußern, auf o,56 ansteigt. Dieses Ergebnis stützt den Befund von Vonbun/Schönbach (2014, 204 ff.), die ein gesteigertes politisches Interesse ebenfalls mit einer gesteigerten aktiven und passiven Online-Nutzung politischer Inhalte in Verbindung bringen konnten. Es zeigt allerdings auch, dass die Verfügbarkeit politischer Informationen über soziale Netzwerke nicht zwangsläufig für eine weitere Verbreiterung der Inhalte über alle Bevölkerungsgruppen hinweg sorgt. Gerade Personen, die sich für das politische Geschehen interessieren, suchen über diese Kanäle auch aktiv danach.

\footnotetext{
5 Das Odds Ratio der Altersvariable ist kleiner o(o,96 in Modell I bzw. o,97 in Modell III). Ein direkter Vergleich der Effektgröße mit jenen Werten über I ist problematisch, da der Wertebereich von Odds Ratios von o bis $+\infty$ definiert und daher stark asymmetrisch ist. Effektgrößen kleiner I können durch eine einfache Rechenoperation, bei der der Wert I durch den errechneten Odds Ratio dividiert wird, vergleichbar gemacht werden. Die Odds der Nutzung der sozialen Netzwerke entsprechen dem Faktor I,O4 (Modell I) beziehungsweise I,O3 (Modell III). Die Chancen, dass eine Person soziale Plattformen zur Informationsgewinnung nützt, steigen mit zunehmender Bildung um den Faktor I,I6 an (Modell III in Tabelle I)

6 Die Berechnung der vorhergesagten Wahrscheinlichkeiten zur politischen Nutzung sozialer Plattformen basiert auf Modell III. Wir betrachten dabei die Nutzungswahrscheinlichkeit, wenn wir den Wert einer Variable variieren (beispielsweise das Alter) und alle anderen Variablen auf ihrem durchschnittlichen Wert konstant halten. Als durchschnittliche Werte nehmen wir den Modus bei kategorialen Variablen und den Mean bei kontinuierlichen Variablen.
} 
Tabelle 1: Nutzung sozialer Netzwerke zur politischen Information

\begin{tabular}{|c|c|c|c|c|c|c|}
\hline \multirow[b]{2}{*}{ Alter in Jahren } & \multicolumn{2}{|c|}{$\begin{array}{l}\text { Modell I: } \\
\text { Akteur }\end{array}$} & \multicolumn{2}{|c|}{$\begin{array}{l}\text { Modell II: } \\
\text { Vertrauen }\end{array}$} & \multicolumn{2}{|c|}{$\begin{array}{l}\text { Modell III: } \\
\text { Kombiniert }\end{array}$} \\
\hline & $0,956^{* * *}$ & $(-8,95)$ & & & $0,971^{\star \star \star}$ & $(-4,93)$ \\
\hline \multicolumn{7}{|l|}{ formaler Bildungsgrad } \\
\hline Pflichtschule & \multicolumn{2}{|c|}{ Referenzkategorie } & & & \multicolumn{2}{|c|}{ Referenzkategorie } \\
\hline Lehre & 1,404 & $(1,62)$ & & & $1,759 * *$ & $(2,16)$ \\
\hline BMS/Fachschule & $1,569^{*}$ & $(1,78)$ & & & 1,433 & $(1,18)$ \\
\hline Matura & 1,362 & $(1,51)$ & & & $1,498^{*}$ & $(1,67)$ \\
\hline Universität & $1,786^{\star \star}$ & $(2,33)$ & & & $2,232^{\star * *}$ & $(2,64)$ \\
\hline Frau & 0,968 & $(-0,22)$ & & & 1,075 & $(0,39)$ \\
\hline Interesse an der Politik & $1,635^{\star * *}$ & $(4,25)$ & & & $1,526^{* * *}$ & $(2,95)$ \\
\hline Politisch Extrem & $1,163^{* * *}$ & $(2,59)$ & & & 1,030 & $(0,40)$ \\
\hline Vertrauen in traditionelle Medien & & & $0,807^{\star \star *}$ & $(-3,47)$ & $0,792^{\star * *}$ & $(-3,52)$ \\
\hline Vertrauen in Informationen aus dem Internet & & & 1,193 & $(1,24)$ & 1,217 & $(1,34)$ \\
\hline \multicolumn{7}{|l|}{ Erfahrung mit Fake News } \\
\hline ja, sehr oft & & & \multicolumn{2}{|c|}{ Referenzkategorie } & \multicolumn{2}{|c|}{ Referenzkategorie } \\
\hline ja,manchmal & & & $0,605^{\star *}$ & $(-2,27)$ & 0,764 & $(-1,15)$ \\
\hline ja, aber nur vereinzelt & & & $0,451^{\star * *}$ & $(-3,27)$ & $0,563^{* *}$ & $(-2,27)$ \\
\hline nein, nie & & & $0,329 * * *$ & $(-3,23)$ & $0,433^{* *}$ & $(-2,28)$ \\
\hline$N$ & \multicolumn{2}{|c|}{1.151} & \multicolumn{2}{|c|}{675} & \multicolumn{2}{|c|}{675} \\
\hline pseudo $R^{2}$ & \multicolumn{2}{|c|}{0,093} & \multicolumn{2}{|c|}{0,036} & \multicolumn{2}{|c|}{0,082} \\
\hline Adjusted Count R2 & \multicolumn{2}{|c|}{0,08} & \multicolumn{2}{|c|}{0,13} & \multicolumn{2}{|c|}{0,22} \\
\hline
\end{tabular}

Schließlich ging Hypothese 3 davon aus, dass Personen, die sich auf einer politischen Links-Rechts-Skala näher an den Rändern einordnen, eher Informationen über soziale Netzwerke suchen als Personen, die sich in der Mitte einordnen. Dies wird im kleineren Modell I des Akteurs bestätigt. Hier weist die Variable Politisch Extrem einen signifikanten Wert größer I auf, der ausdrückt, dass eine zunehmende Orientierung am Rand der Skala mit einer höheren Chance der Nutzung von Twitter und ähnlichen Plattformen zur politischen Informationssuche einhergeht. Modell III, das auf dem kleineren Sample an Personen basiert, die angaben, auf sozialen Netzwerken zumindest zufällig mit politischen Infor- mationen in Kontakt zu kommen, zeigt diesen Effekt nicht. Berechnet man ein weiteres Modell ohne der Variablen Vertrauen in traditionelle Medien und dafür mit allen befragten Personen, dann bleibt der signifikante Effekt der Variablen Politisch Extrem bestehen (siehe Anhang C). Wir sehen die Hypothese 3 daher als bestätigt an. Dies verdeutlicht auch nochmals die folgende Abbildung I. Bei Personen, die sich in der Mitte des politischen Spektrums verorten, liegt die Wahrscheinlichkeit soziale Plattformen zur Informationsgewinnung $\mathrm{zu}$ besuchen bei o,28. Dieser Wert steigt auf o,4I bei Personen, die sich selbst an den Rändern einer Links-Rechts-Skala einstufen. 
Abbildung 1: Wahrscheinlichkeit der politischen Social Media-Nutzung I

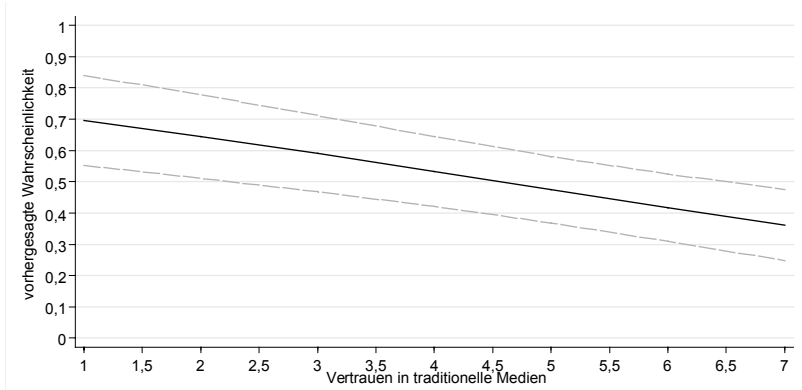

Anmerkung: Die Berechnung der vorhergesagten Wahrscheinlichkeiten und der entsprechenden 95\%-Konfidenzintervalle basiert auf Modell III ohne der Variablen Vertrauen in traditionelle Medien. Die übrigen Variablen wurden auf ihrem Modus (kategoriale Variablen) beziehungsweise ihrem Mean (kontinuierliche Variablen) konstant gehalten. Zur Erinnerung: Die Politisch Extrem-Skala basiert auf der Links-Rechts-Skala. Personen, die auf der Links-Rechts-Skala die Werte 5 oder 6 angegeben haben, besitzen in der Grafik den Wert 1. Personen, die auf der LinksRechts-Skala die Werte 1 oder 10 angegeben haben, besitzen in der Grafik den Wert 5.

In diesem Zusammenhang lohnt sich auch ein Blick auf die deskriptive Verteilung der ursprünglichen Antworten auf der zehnstufigen Links-Rechts-Skala. Personen, die sich jeweils auf den äußeren beiden Polen ganz links oder ganz rechts einordnen, verwenden zu 39 beziehungsweise zu 46 Prozent soziale Plattformen zur politischen Information. Es zeigt sich daher ein deutlicher Unterschied, an welchem Endpunkt der Skala eine Person zu verorten ist. Der Effekt kommt stärker von einer rechten extremen Position als von einer linken. Dieses Ergebnis basiert allerdings nur auf 7I (extrem linke Positionen) beziehungsweise 67 (extrem rechte Positionen) Personen, die sich diesen Gruppen zugeordnet haben. Im Vergleich dazu nützen von den 409 befragten Personen, die sich in der Mitte der Skala einstufen, nur 25 Prozent soziale Plattformen zur politischen Information. Dies spiegelt teilweise die Ergebnisse der vorherigen Arbeit zur politischen Online-Kommunikation in Österreich wider (Vonbun/Schönbach 20I4, 204f). Während die AutorInnen keinen Effekt auf die zumindest einmal im Jahr aktive oder passive politische Online-Kommunikation feststellen konnten, zeigte sich ein Effekt bei der Häufigkeit der Nutzung. Personen, die politisch stark rechts orientiert sind, verwenden häufiger passive politische Online-Kommunikation zur Information. Für politisch stark links orientierte Personen trifft dies nicht zu.

Rolle von Vertrauen bei der politischen Social MediaNutzung

Nach der Beschreibung der Effekte aus der Sicht des Akteurs werden nun die Hypothesen, die die Rolle von Vertrauen bei der Nutzung sozialer Medien zur politischen
Informationssuche thematisiert haben, diskutiert. Hypothese 4 hat das Vertrauen in die traditionellen Medien als Erklärungsfaktor herangezogen und argumentiert, dass ein geringes Vertrauen in diese Kanäle die Wahrscheinlichkeit der Nutzung von sozialen Plattformen steigen lässt. Die Modelle können diese Annahme bestätigen. Mit sinkendem Vertrauen steigen die Chancen, dass soziale Medien zur Information genutzt werden.

Abbildung 2: Wahrscheinlichkeit der politischen Social Media-Nutzung II

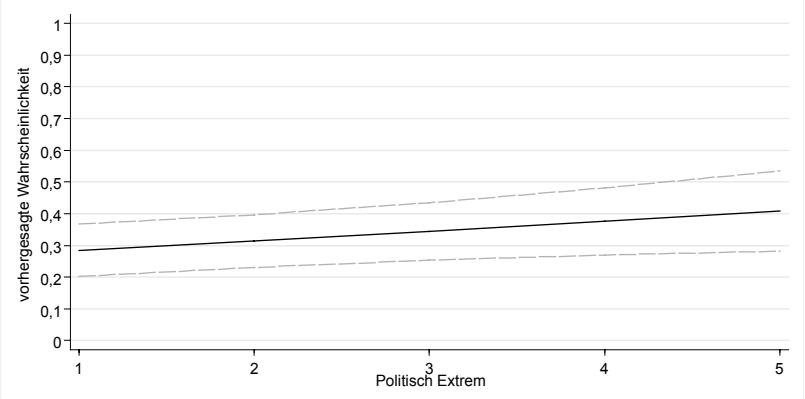

Anmerkung: Die Berechnung der vorhergesagten Wahrscheinlichkeiten und der entsprechenden 95\%-Konfidenzintervalle basiert auf Modelle III (Tab. 1). Die übrigen Variablen wurden auf ihrem Modus (kategoriale Variablen) beziehungsweise ihrem Mean (kontinuierliche Variablen) konstant gehalten.

Abbildung 2 berechnet erneut die vorhergesagten Wahrscheinlichkeiten, dass Personen soziale Medien zur politischen Information verwenden. Die X-Achse zeigt nun die Veränderung des Vertrauens in die traditionellen Medien Tageszeitungen, Fernsehen und Radio an. Es zeigt sich, dass die Wahrscheinlichkeit ein/e UserIn von sozialen Netzwerken zu sein, bei geringem Vertrauen (Wert I) auf der 7-teiligen Skala bei o,7 liegt und bis auf 0,36 am anderen Endpunkt (Wert 7) absinkt.

Hypothese 5 wird nicht bestätigt. Die Nutzung sozialer Plattformen zur Information scheint nicht durch das Vertrauen, welches eine befragte Person dem Internet entgegenbringt, beeinflusst zu werden. Die Odds Ratios sind zwar größer I, die Effekte sind allerdings in keinem der beiden getesteten Modelle signifikant.

Besonders interessant ist auch der Befund in Bezug auf Hypothese 6. Diese postulierte, dass Personen ohne bewusste Erfahrungen mit Fake News eher soziale Netzwerke zur politischen Information nützen. Das Gegenteil ist jedoch der Fall. Je häufiger solche Erfahrungen gemacht werden, umso größer die Chancen, dass Social Media Teil der eigenen Informationsstrategie sind. Die Odds Ratios sind niedriger als I und fast ausnahmslos signifikant. Dieser zunächst paradox klingende Befund lässt sich vermutlich dadurch erklären, dass erst die häufigere Nutzung sozialer Netzwerke zu Informationszwecken überhaupt zu einer (gehäuften) Erfahrung mit Falschnachrichten führen. So gesehen scheinen Fake 
News ein Teil der Online-Nachrichtenpräsenz zu sein und die Ergebnisse deuten nicht darauf hin, dass sich die NutzerInnen aufgrund schlechter Erfahrungen auf sozialen Plattformen von diesem Medium abwenden.

Tatsächlich gab in der zugrunde liegenden Umfrage gut ein Viertel der befragten Personen, die soziale Medien zur Information nützen, an, sehr oft Erfahrungen mit Fake News zu machen. Weitere 40 Prozent beziehungsweise 25 Prozent stoßen manchmal beziehungsweise vereinzelt auf Falschmeldungen und nur 9 Prozent meinen, nie mit unwahren Aussagen konfrontiert worden zu sein (siehe Anhang D). Im gesamten Sample sinken die Erfahrungswerte mit Fake News. Dies liegt, wie auch die multivariaten Ergebnisse vermuten lassen, an der fehlenden Gelegenheit, bewusst mit Falschnachrichten in Kontakt zu kommen.

Abbildung 3 veranschaulicht wiederum die Stärke des Effekts. Die Abbildung zeigt die vorhergesagte Wahrscheinlichkeit, dass eine Person soziale Plattformen zur Information verwendet, in Abhängigkeit ihrer Erfahrung mit Fake News. Die Wahrscheinlichkeit, ein/e UserIn zu sein, liegt bei Personen, die noch keine konkreten Erfahrungen mit Falschaussagen gemacht haben, bei o,37 und steigt bei Personen, die oft mit der vermeintlichen Unwahrheit konfrontiert werden, auf 0,58 an.

Neben der Interpretation der einzelnen Koeffizienten ist schließlich auch die Güte des gesamten Modells wesentlich für eine vollständige Interpretation der Ergebnisse. Die häufig angeführte Maßzahl Pseudo R2 zur Einschätzung der Modellgüte logistischer Regressionsmodelle ist unseres Erachtens kein sehr intuitives Maß (vgl. auch Kohler und Kreuter 2008, 278 ff.). Daher geben wir in Tabelle I zusätzlich das sogenannte Adjusted Count R2 an. Das Adjusted Count R2 gibt den Anteil durch das Modell korrekt vorhergesagter Fälle in die Kategorien keine Nutzung sozialer Medien zur politischen Information vs. Nutzung sozialer Medien zur politischen Information an, wobei der Anteil der Modalkategorie - der besten Schätzung, wenn keine weitere Kenntnis der unabhängigen Variablen vorliegt - bereits abgezogen wird. Je höher das Adjusted Count R2 im Vergleich zur Modalkategorie, umso mehr verbessern die Modelle die Zuordnung der Fälle in die richtige Kategorie. Modell I verbessert die Zuordnung nur um rund acht Prozentpunkte. Die Modelle mit der stärkeren Erklärungskraft sind die Modelle II (rund I3 Prozentpunkte) und III (rund 22 Prozentpunkte). Daraus folgt, dass insbesondere das Vertrauen Aufschluss über die Nutzung sozialer Medien zur politischen Information liefern und eine Kombination beider Erklärungsgruppen den größten Wert aufweist.

\section{Schluss}

Aus der Analyse der vorliegenden Daten lassen sich zusammenfassend mehrere Befunde und weiterführende Überlegungen ableiten. Zunächst kommt dem Alter immer noch eine zentrale Rolle zu, wenn es darum geht, die

Abbildung 3: Wahrscheinlichkeit der politischen Social Media-Nutzung III

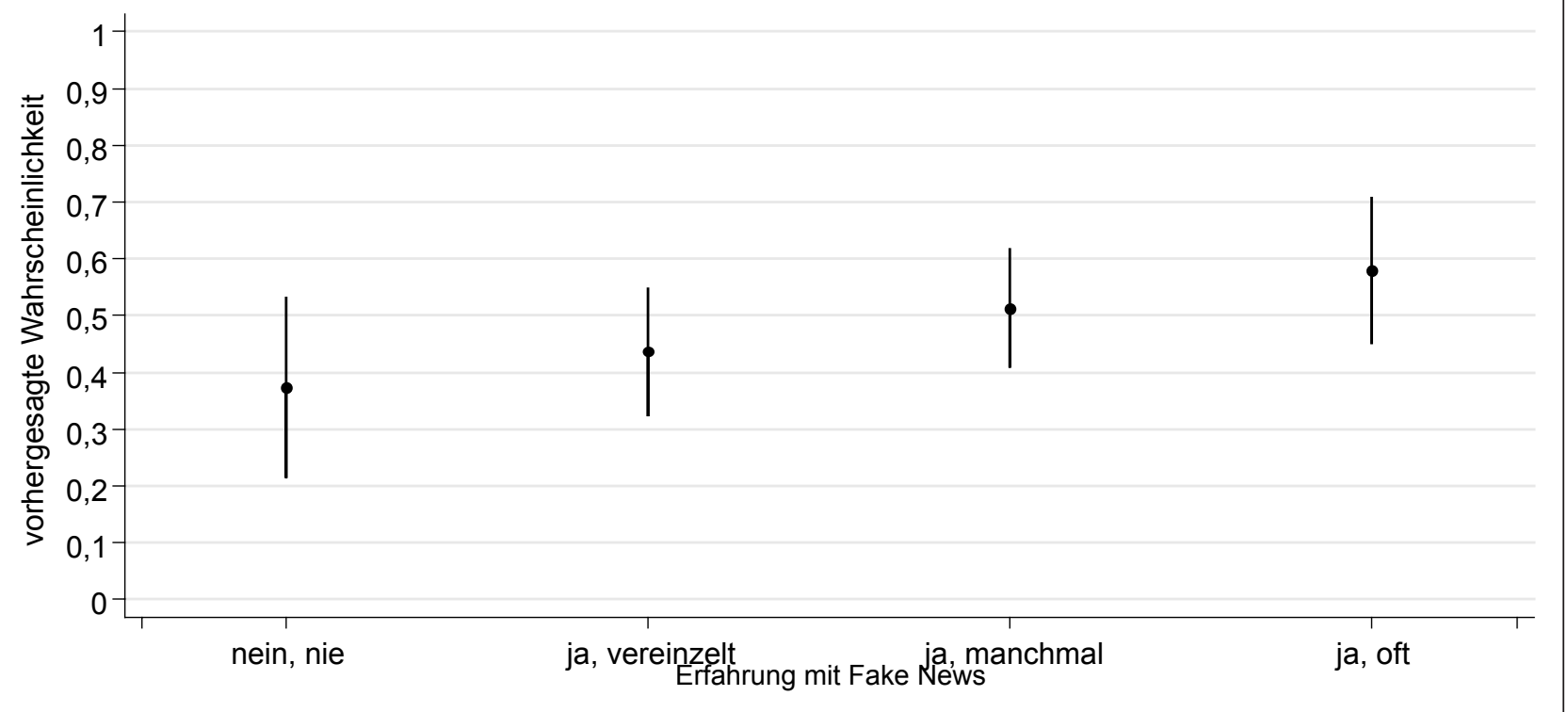

Anmerkung: Die Berechnung der vorhergesagten Wahrscheinlichkeiten und der entsprechenden 95\%-Konfidenzintervalle basiert auf Modell III (Tab.1). Die übrigen Variablen wurden auf ihrem Modus (kategoriale Variablen) beziehungsweise ihrem Mean (kontinuierliche Variablen) konstant gehalten. 
NutzerInnen sozialer Netzwerke zu beschreiben. Es gibt damit eine Kluft im politischen Informationsverhalten zwischen jüngeren und älteren Personen, was die Frage aufwirft, ob dadurch eine gemeinsame Informationsbasis eingeschränkt oder im Extremfall verunmöglicht wird. Dass dies auch konkret politische Auswirkungen hätte, liegt auf der Hand und wäre es durchaus Wert, näher erforscht zu werden.

Ein weiteres Ungleichgewicht ergibt sich durch den Faktor politisches Interesse, der ebenfalls eine gewisse Erklärungskraft aufweist. Personen, die sich selbst als interessierter bezeichnen, nennen Social Media vermehrt als eine ihrer Quellen für politische Information. Letztere vergrößern so gesehen in erster Linie den Informationspool jener, die sich bereits mit Politik auseinandersetzen. Allerdings ist aus den Daten nicht ablesbar, welchen Einfluss die Verfügbarkeit von neuen OnlineQuellen auf die grundsätzliche Hinwendung zu politischen Informationen hat, sprich ob Informationsangebote in sozialen Netzwerken neue Personen ansprechen oder nicht.

Der in der Analyse gezeigte Effekt des Vertrauens in traditionelle Medien deutet darauf hin, dass Social Media ihren NutzerInnen etwas bieten, was sie als attraktive Alternative zu Fernsehen, Radio und Tageszeitungen erscheinen lassen. Ob das sich selbst bestätigende Informationen im Sinne von Phänomenen wie Filterblasen bzw. Echokammern sind oder andere Aspekte Vorrang haben, lässt sich nicht sagen, wenngleich das Ergebnis in diese Richtung interpretiert werden kann.

Speziell für diesen, aber auch die anderen beiden genannten Punkte wäre es in weiteren Untersuchungen wichtig, eine Differenzierung der konkret genutzten Inhalte in sozialen Netzwerken vorzunehmen. Es macht einen Unterschied, ob jemand Beiträge traditioneller Medien lieber in Social Media-Form konsumiert bzw. dort damit in Kontakt kommt, oder ob er/sie sich gänzlich anderen Quellen zuwendet. Dies scheint vor allem deshalb für weitere Untersuchungen relevant, weil die Folgen für die Öffentlichkeit an sich spürbar sind und schon jetzt offensichtlich ist, dass sich das Zusammenspiel der einzelnen Akteure in der Bevölkerung sowie die Kommunikation generell verändern (vgl. Katzenbach 20I6, 3), weg von einem reinen Medienraum mit Inhalten hin zu einem sich ständig verändernden Prozess (vgl. Katzenbach 2016, I6 f.). Wenn ForscherInnen wie Fenton Recht haben und die beobachteten politischen Effekte durch die vermehrte Social Media-Nutzung immer wieder auch damit zu tun haben, dass sich BürgerInnen politisch machtlos fühlen (vgl. Fenton 20I6, 354) und diese Machtlosigkeit auf Facebook oder Twitter als Protest und Radikalisierung sichtbar wird, ist es auch für das gesellschaftliche Zusammenleben in Österreich wesentlich, genauer hinzuschauen.

\section{Literatur}

Aarts, Kees/Holli A. Semetko (2003), The Divided Electorate: Media Use and Political Involvement, in: The Journal of Politics, Vol. 65(3), 759-784.

Allcott, Hunt/Matthew Gentzkow (2017), Social Media and Fake News in the 2016 Election, in: Journal of Economic Perspectives, Vol. 3I(2), 2II-236.

Barberá, Pablo (2015), How Social Media Reduces Mass Political Polarization. Evidence from Germany, Spain, and the U.S., Konferenzbeitrag, Amercian Political Science Association Annual Meeting, 3.6.9.2015, San Francisco.

Barberá, Pablo/John T. Jost/Jonathan Nagler/Joshua A. Tucker/Richard Bonneau (2015), Tweeting From Left to Right: Is Political online Communication More Than an Echo Chamber?, in: Psychological Science, Vol. 26(IO), I53I-I542.

Barthes, Roland (1989), Die helle Kammer, Frankfurt am Main: Suhrkamp.

Baudrillard, Jean (1983), Lasst euch nicht verführen, Berlin: Merve.

Baudrillard, Jean (2003), Requiem for the Media, in: Wardrip-Fruin, Noah/Nick Montfort (Hg.), The New Media Reader, Cambridge: MIT Press, 277-288.

Boxell, Levi/Matthew Gentzkow/Jesse M. Shapiro (2017), Greater Internet use is not associated with faster growth in political polarization among US demographic groups, in: Proceedings of the National Academy of Sciences, Vol. II4(40), I06I2-IO6I7.

Chang, Jung-Hua/Yu-Qian Zhu/Shan-Huei Wang/Yi-Jung Li (2018), Would you change your mind? An empirical study of social impact on Facebook, in: Telematics and Informatics, Vol. 35(I), 282-292.

Coyne, Richard (200I), Technoromanticism. Digital narrative, holism, and the romance of the real. Cambridge: MIT Press.

Colleoni, Elanor/Alessandro Rozza/Adam Arvidsson (2014), Echo Chamber or Public Sphere? Predicting Political Orientation and Measuring Political Homophily in Twitter Using Big Data, in: Journal of Communication, Vol. 64(2), 317-332.

Dickel, Sascha/Jan-Felix Schrape (2015), Dezentralisierung, Demokratisierung, Emanzipation. Zur Architektur des digitalen Technikutopismus, in: Leviathan, Vol. 43(3), 442-463.

Diehl, Trevor/ Brian E. Weeks/Homero Gil de Zuñiga (2015), Political persuasion on social media: Tracing direct and indirect effects of news use and social interaction, in: New Media \& Society, Vol. I8(9), I875-I895.

Downs, Anthony (1957), An Economic Theory of Democracy, New York: Harper \& Brothers.

Dubois, Elizabeth/Grant Blank (2018), The echo chamber is overstated: the moderating effect of political inte- 
rest and diverse media, in: Information, Communication \& Society, Vol. $2 \mathrm{I}(5), 729-745$.

Emmer, Martin/ Gerhard Vowe/Jens Wolling (20II), Bürger online. Die Entwicklung der politischen OnlineKommunikation in Deutschland, Konstanz: UVK.

Europäische Kommission (20I7), Media Use in the European Union, Internet: https://ec.europa.eu/commfrontoffice/publicopinion/index.cfm/ResultDoc/download/ DocumentKy/79405 (Zugriff: 03.05.2018).

Eurostat (2018), People participating in social networks, Internet: http://ec.europa.eu/eurostat/cache/infographs/ict/bloc-Ib.html (Zugriff: 03.05.2018).

Faas, Thorsten/ Benjamin C. Sack (Hg.) (2016), Politische Kommunikation in Zeiten von Social Media, Bonn: Bonner Akademie für Forschung und Lehre praktischer Politik.

Fairclough, Norman (200I), Language and Power. Marlow: Longman.

Fenton, Natalie (2016), Left out? Digital media, radical politics and social change, in: Information, Communication \& Society, Vol. I9(3), 346-36I.

Flusser, Vilém (1987), Die Schrift. Hat Schreiben Zukunft?, Göttingen: Immatrix Publications.

Hagen, Lutz M./ Anne-Marie in der Au/Mareike Wieland (20I7a), Polarisierung im Social Web und der intervenierende Effekt von Bildung: eine Untersuchung zu den Folgen algorithmischer Medien am Beispiel der Zustimmung zu Merkels „Wir schaffen das!“, in: kommunikation@gesellschaft, Vol. I8, I-20.

Hagen, Lutz M./ Anne-Marie in der Au/Mareike Wieland (20I7b), Algorithmischer Strukturwandel der Öffentlichkeit. Wie die automatische Selektion im Social Web die politische Kommunikation verändert und welche Gefahren dies birgt, in: MedienJournal, Vol. 4I(2), I27-I 43.

Hughes, David John/Moss Rowe/Mark Batey/ Andrew Lee (2012), A tale of two sites: Twitter vs. Facebook and the personality predictors of social media usage, in: Computers in Human Behavior, Vol. 28(2), 56I-569.

Institut für Strategieanalysen (ISA, 20I7), Digitalmonitor. Studie im Auftrag des Staatssekretariats für Diversität, öffentlichen Dienst und Digitalisierung. Wien.

Integral (20I7), Austrian Internet Monitor, 3/20I7, Internet: http://www.integral.co.at/de/aim/ (Zugriff: 03.05.2018).

Johnson, Sally/Tommaso M. Milani (2010), Language Ideologies and Media Discourse: Texts, Practices, Politics. London/NY: continuum books.

Jouët, Josiane/Thierry Vedel/Jean-Baptiste Comby (20II), Political information and interpersonal conversations in a multimedia environment: A quantitative and qualitative examination of information practices in France, European Journal of Communication 26(4), 36I-375.
Katzenbach, Christian (2016), Von kleinen Gesprächen zu großen Öffentlichkeiten? Zur Dynamik und Theorie von Öffentlichkeiten in sozialen Medien, in: Klaus, Elisabeth/ Ricarda Drüeke (Hg.), Öffentlichkeiten und gesellschaftliche Aushandlungsprozesse: theoretische Perspektiven und empirische Befunde, Bielefeld: transcript Verlag, I5I-I74.

Kneuer, Marianne/Samuel Salzborn (2016), Digitale Medien und ihre Wirkung auf demokratische Prozesse, in: Zeitschrift für Vergleichende Politikwissenschaft, Vol. IO(2), I-I4.

Kohler, Ulrich/Frauke Kreuter (2008), Datenanalyse mit Stata: Allgemeine Konzepte der Datenanalyse und ihre praktische Anwendung, München: Oldenbourg Wissenschaftsverlag, 3. Auflage.

meinungsraum.at: Online Panel Österreich, Internet: https://meinungsraum.at/online-panel-osterreich/ (Zugriff: 03.05.2018).

Östman, Johan (2012), Information, expression, participation: How involvement in user- generated content relates to democratic engagement among young people, in: New Media \& Society, I4(6), IOO4-IO2I.

Pariser, Eli (2012), Filter Bubble: Wie wir im Internet entmündigt werden. München: Carl Hanser Verlag $\mathrm{GmbH} \&$ Co. KG.

Plasser, Fritz/Günther Lengauer (2012), Rules of the Game: Österreichs politische Kommunikationskultur im europäischen Vergleich, in: Plasser, Fritz (Hg.), Erfolgreich wahlkämpfen: Massenmedien und Wahlkampagnen in Österreich, Wien: facultas.wuv, 29-55.

Reuters Institute (2018), Digital News Report 20I7, Internet: https://reutersinstitute.politics.ox.ac. uk/sites/default/files/Digital\%2oNews \% 20 Report\%2020I $7 \% 2$ oweb_o.pdf?utm _ source $=$ digitalnews report.org \&ut m medium=referral (Zugriff: 03.05.2018).

Sarcinelli, Ulrich (Hg.) (I998), Politikvermittlung und Demokratie in der Mediengesellschaft: Beiträge zur politischen Kommunikationskultur. Opladen/Wiesbaden: VS Verlag für Sozialwissenschaften.

Schrape, Jan-Felix (2015), Social Media, Massenmedien und Öffentlichkeit - eine soziologische Einordnung, in: Blum, Roger/Heinz Bonfadelli/Kurt Imhof/Otfried Jarren/Vinzenz Wyss (Hg.), Demokratisierung durch Social Media? Mediensymposium 2012, Wiesbaden: Springer VS, I99-2I2.

Sheldon, Pavica/Katherine Bryant (2016), Instagram: Motives for its use and relationship to narcissism and contextual age, in: Computers in Human Behavior, Vol. 58, 89-97.

Statistik Austria (2018a), IKT-Einsatz in Haushalten, Internet: https:/www.statistik.at/web_de/statistiken/ energie_umwelt_innovation_mobilitaet/informa- 
tionsgesellschaft/ikt-einsatz_in_haushalten/index. html (Zugriff: 03.05.2018).

Statistik Austria (2018b), IKT-Einsatz in Haushalten 20I7, Internet: https://www.statistik.at/web_de/statistiken/energie_umwelt_innovation_mobilitaet/ informationsgesellschaft/ikt-einsatz_in_haushalten/O73636.html (Zugriff: 03.05.2018).

Vonbun, Ramona/ Klaus Schönbach (2014), Wer ist politisch aktiv im Social Web? Eine Studie zur politischen Online-Kommunikation in Österreich, in: $P u^{-}$ blizistik, Vol. 59, 199-212.

Wolling, Jens (2016), Struktureller Wandel der politischen Kommunikation durch die Diffusion von Online-Medien: empirischer Test einer weitreichenden These, in: Henn, Philipp/Dennis Frieß (Hg.), Politische Online-Kommunikation: Voraussetzungen und Folgen des strukturellen Wandels der politischen Kommunikation, 19-45.

\section{Autoren}

Dr. Katrin Praprotnik ist wissenschaftliche Mitarbeiterin der Donau-Universität Krems und leitet das Austrian Democracy Lab. Ihre Forschungsschwerpunkte umfassen insbesondere das politische System Österreich, Parteien und Wahlen im europäischen Vergleich sowie Repräsentationsforschung. Praprotnik publizierte unter anderem in den Fachzeitschriften West European Politics, American Journal of Political Science und Electoral Studies.

Dr. Flooh Perlot arbeitet als wissenschaftlicher Mitarbeiter (Politikwissenschaft) an der Karl-Franzens-Universität Graz und ist dort in erster Linie für die Datenerhebungen und Auswertungen des Demokratieradars zuständig. Seine Arbeitsschwerpunkte umfassen Politik und Medien, Internet und Demokratie, Wahlforschung sowie Datenvisualisierung.

Dr. Daniela Ingruber ist Politikwissenschafterin, Medientheoretikerin, Demokratie- und Kriegsforscherin und als wissenschaftliche Mitarbeiterin an der DonauUniversität Krems tätig. Arbeitsschwerpunkte sind Demokratietheorie, Partizipation, politische Philosophie, Konflikttransformation, Film und ethischer Journalismus.

Univ-Prof. Dr. Peter Filzmaier ist Professor für Politikwissenschaft an der Karl-Franzens-Universität Graz und der Donau-Universität Krems sowie geschäftsführender Gesellschafter des Instituts für Strategieanalysen (ISA) in Wien. Arbeitsschwerpunkte sind Politik- und Wahlanalysen, Politische Kommunikation, Politik und Medien bzw. Internet und Demokratie sowie Vergleich politischer Systeme (insbesondere politisches System und politischer Prozess in den USA). 


\section{Anhang A. Struktur Grundgesamtheit und Stichprobe}

\begin{tabular}{|c|c|c|c|}
\hline & $\begin{array}{r}\text { Stichprobe (absolut, } \\
\text { ungewichtet) }\end{array}$ & $\begin{array}{r}\text { Stichprobe (in Prozent, } \\
\text { gewichtet) }\end{array}$ & Grundgesamtheit (in Prozent) \\
\hline Männer & 566 & 50 & 51 \\
\hline Frauen & 579 & 50 & 49 \\
\hline keine Matura & 787 & 73 & 69 \\
\hline Matura oder höher & 358 & 27 & 31 \\
\hline $16-24$ & 334 & 15 & 17 \\
\hline $25-34$ & 200 & 17 & 22 \\
\hline $35-44$ & 198 & 23 & 21 \\
\hline $45-54$ & 222 & 25 & 24 \\
\hline $55-64$ & 172 & 20 & 17 \\
\hline
\end{tabular}

Anmerkung: Angaben in Prozent, Rest auf 100=Rundungsfehler; nur Befragte ab 16 Jahren, restliche Fälle in der Stichprobe entfallen auf Personen unter 14 bzw. ab 65 Jahren, die aufgrund der mangelnden Vergleichsmöglichkeit hier nicht dargestellt sind.

Quelle: ISA 2017; Statistik Austria 2018b; Integral 2017 (Bildung). 


\section{Anhang B. Fragen im Originalwortlaut}

\section{Abhängige Variable}

Variable: Nutzung sozialer Netzwerke zur politischen Information

Woher beziehen Sie Ihre Informationen über politische Themen in Österreich?

(Fernsehen/Tageszeitungen/Internet, aber keine sozialen Netzwerke/Radio/soziale Netzwerke im Internet/ Sonstiges)

Personen, die bereits angegeben haben, sich eher nicht für politische Themen zu interessieren wurde die Frage mit folgendem Zusatz gestellt: „Wenn Sie sich doch einmal informieren möchten, ...“

Unabhängige Variablen

Variable: Alter in Jahren

Wie alt sind Sie?

Variable: formaler Bildungsgrad

Ihre höchste abgeschlossene Schulbildung?

Variable: Frau

Ihr Geschlecht?

Variable: Interesse an der Politik

Interessieren Sie sich für politische Themen in Österreich?

(ja, sehr/ja, eher schon/nein, eher nicht/nein, gar nicht)

Variable: Politisch Extrem

Zum Schluss, in der Politik spricht man ja immer wieder von links und rechts. Auf einer Skala von I bis Io, wo würden Sie sich bei diesen Begriffen zuordnen? I bedeutet sehr weit links, Io bedeutet sehr weit rechts.

Variable: Vertrauen in traditionelle Medien

Vergleichen Sie nun bitte die „normalen“ Medien mit den sozialen Netzwerken, die Sie nutzen: Wenn Sie sich über politische Themen in Österreich informieren, wer bietet da eher... glaubwürdige Informationen?

- $\quad$ soziale Netzwerke, die Sie nutzen (I) - Zeitungen und Zeitschriften, die Sie nutzen (7)

- $\quad$ soziale Netzwerke, die Sie nutzen(I) - Fernsehnachrichten, die Sie nutzen (7)

- $\quad$ soziale Netzwerke, die Sie nutzen (I) - Radionachrichten, die Sie nutzen (7)

Variable: Vertrauen in Informationen aus dem Internet

Wenn Sie einmal an die Informationen und Nachrichten denken, die Sie online beziehen, inwieweit stimmen Sie da folgenden Aussagen zu?

Informationen aus dem Internet kann man oft nicht vertrauen.

Ich mache mir häufig Gedanken darüber, ob Informationen aus dem Internet stimmen.

(stimme sehr zu/stimme eher zu/stimme eher nicht zu/stimme gar nicht zu)

Variable: Erfahrung mit Fake News

Sind Ihnen selbst solche „Fake News“ schon einmal untergekommen?

(ja, sehr oft/ja, manchmal/ja, aber nur vereinzelt/nein, nie) 


\section{Anhang C. Variante von Modell III}

Modell III: Kombiniert

(ohne Vertrauen in traditionelle Medien)

Alter in Jahren

$0,962^{* * *}$

$(-7,60)$

formaler Bildungsgrad

...Pflichtschule

...Lehre

1,379

$(1,49)$

...BMS/ Fachschule

1,443

$(1,42)$

...Matura

1,367

$(1,51)$

...Universität

$1,553 *$

Frau

1,031

Interesse an der Politik

$1,552^{\star \star \star}$

Politisch Extrem

$1,148^{* *}$

Vertrauen in Internet

$1,245^{*}$

Erfahrung mit Fake News

...ja, sehr oft

...ja, manchmal

$0,579 * * *$

...ja, aber nur vereinzelt

$0,457^{* * *}$

...nein, nie

$0,215^{* * *}$

$N$ 1.151

pseudo $R^{2}$

0,119

* $p<0,1$;* $p<0,05 ; p$ *** $<0,01$

Anmerkung: binäres Logit-Modell. Effektgrößen sind Odds Ratios (z-Statistik). 
Anhang D. Erfahrungen mit Fake News unter Personen, die soziale Medien bewusst zur politischen Information verwenden

\begin{tabular}{llll}
\hline Erfahrung mit Fake News & N & \% & Kum. \% \\
\hline ja, sehr oft & 100 & 26.5 & 26.5 \\
ja, manchmal & 150 & 39.8 & 66.3 \\
ja, aber nur vereinzelt & 94 & 24.3 & 91.3 \\
nein, nie & 33 & 8.8 & 100 \\
\hline gesamt & 377 & 100 & \\
\hline
\end{tabular}

Anmerkung: Ergebnisse summieren sich nicht auf 100 durch Rundung der einzelnen Werte. Kum. \% steht für kumulierte Prozentwerte. 
